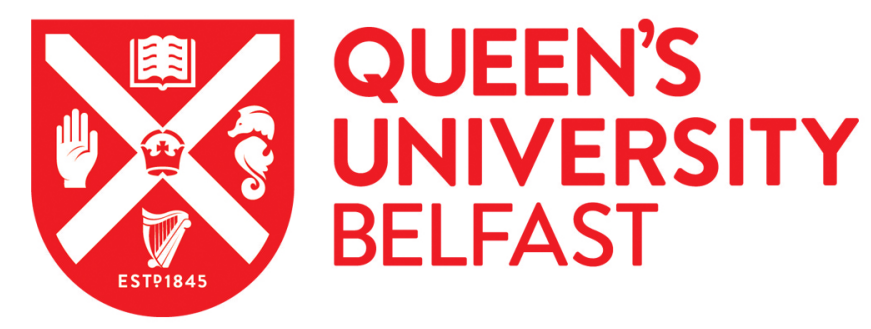

\title{
Conducting Polyetheretherketone Nanocomposites with an Electrophoretically Deposited Bioactive Coating for Bone Tissue Regeneration and Multimodal Therapeutic Applications
}

He, M., Zhu, C., Xu, H., Sun, D., Chen, C., Feng, G., Liu, L., Li, Y., \& Zhang, L. (2020). Conducting

Polyetheretherketone Nanocomposites with an Electrophoretically Deposited Bioactive Coating for Bone Tissue Regeneration and Multimodal Therapeutic Applications. ACS Applied Materials and Interfaces.

https://doi.org/10.1021/acsami.0c20145

\section{Published in:}

ACS Applied Materials and Interfaces

Document Version:

Peer reviewed version

Queen's University Belfast - Research Portal:

Link to publication record in Queen's University Belfast Research Portal

\section{Publisher rights}

(C) 2020 American Chemical Society

This work is made available online in accordance with the publisher's policies. Please refer to any applicable terms of use of the publisher.

\section{General rights}

Copyright for the publications made accessible via the Queen's University Belfast Research Portal is retained by the author(s) and / or other copyright owners and it is a condition of accessing these publications that users recognise and abide by the legal requirements associated with these rights.

Take down policy

The Research Portal is Queen's institutional repository that provides access to Queen's research output. Every effort has been made to ensure that content in the Research Portal does not infringe any person's rights, or applicable UK laws. If you discover content in the

Research Portal that you believe breaches copyright or violates any law, please contact openaccess@qub.ac.uk. 


\section{Conducting PEEK nanocomposites with electrophoretically deposited bioactive coating for bone tissue regeneration and multi-modal therapeutic applications}

Miaomiao He ${ }^{1}$, Ce Zhul ${ }^{1}$ Huan Xu' ${ }^{2}$, Dan Sun ${ }^{3}$, Chen Chen ${ }^{1}$, Ganjun Feng ${ }^{1}$, Limin Liu $^{1}$, Yubao Li ${ }^{1}$, Li Zhang $^{1 *}$

1. Analytical \& Testing Center, Department of Orthopedic Surgery and Orthopedic Research Institute, West China Hospital, Sichuan University, Chengdu 610065, China

2. School of Materials and Physics, China University of Mining and Technology, Daxue Road, No. 1, Xuzhou, Jiangsu, 221116 China

3. Advanced Composite Research Group (ACRG), School of Mechanical and Aerospace Engineering, Queen's University Belfast, Belfast, BT9 5AH, UK

* Corresponding author

E-mail address: nic1976@scu.edu.cn (Li Zhang)

Abstract: The use of polyetheretherketone (PEEK) has grown exponentially in the biomedical field in recent decades due to its outstanding biomechanical properties. However, its lack of bioactivity/osteointegration remains an unresolved issue towards its wide use in orthopedic applications. In this work, graphene nanosheets have been incorporated into PEEK to obtain multifunctional nanocomposites. Due to the formation of electrical percolation network and the $\pi-\pi^{*}$ conjugation between graphene and PEEK, the resulting composites have achieved twelve order of magnitude enhancement in its electrical conductivity, and thereby enabled electrophoretic deposition of bioactive/anti-bacterial coating consisting of 
stearyltrimethylammonium chloride (STAC) modified hydroxyapatite (HA). The coated composite implant shows significant boosting of BMSC cell proliferation in vitro. In addition, the strong photothermal conversion effect of the graphene nanofillers have enabled laser induced heating of our nanocomposite implants, where the temperature of the implant can reach $45{ }^{\circ} \mathrm{C}$ in $150 \mathrm{~s}$. The unique multi-functionality of the implant has also been demonstrated for photothermal applications such as enhancing bacterial eradication and tumor cell inhibition, as well as bone tissue regeneration in vivo. The results suggest the strong potential of our multi-functional implant in bone repair applications as well as multi-modal therapy of challenging bone diseases such as osteosarcoma and osteomyelitis.

Keywords: polyetheretherketone (PEEK); graphene; photothermal therapy; electrophoretic deposition; tumor inhibition.

\section{Introduction}

Polyetheretherketone (PEEK) is a popular thermoplastic material widely used in various engineering applications due to its excellent mechanical properties and stability at high temperatures. With the first implantable grade PEEK being commercialized in 1990s [1], the use of PEEK has since grown exponentially in the biomedical field and has rapidly transformed a large section of the medical devices landscape, particularly in spinal implants. Nowadays, PEEK is the standard biomaterial used across a wide range of hard tissue implants including artificial knee joints [2], spine [3], skull [4], and medical fixtures [5]. However, its lack of bioactivity remains the major limitation in its orthopedic applications. 
In order to improve the bioactivity of PEEK implants, researchers have embedded various bioactive nanomaterials, such as hydroxyapatite (HA) [6] and/or titanium dioxide $\left(\mathrm{TiO}_{2}\right)$ [7] nanoparticles into PEEK to create nanocomposites with improved osteoconductivity in vivo. However, the high viscosity of the composite materials has led to additional challenge in large scale melt-processing, and the ceramic based reinforcement materials can easily agglomerate at higher concentration, which may damage the composites structural integrity and lead to stress concentration that compromise the composites tensile/fatigue/toughness properties [8,9]. Taking account of such limitation, various PEEK surface modification technologies have emerged in recent decades, aiming to retain the excellent bulk mechanical properties of PEEK. Lu et al. [10] embedded $\mathrm{Ta}_{2} \mathrm{O}_{5}$ nanoparticles in the near surface of PEEK through plasma immersion ion implantation, and the modified PEEK surface showed significantly improved osteointegration performance. Wen et al. [11] introduced bioactive silicate coating onto PEEK surface using electron beam evaporation and demonstrated the coating's improved osseointegration under osteoporotic condition. Zhu et al. [12] deployed oxygen plasma treatment and poly(dopamine) coating with grafted tripeptide Arg-Gly-Asp to achieve modified PEEK surface with enhanced bioactivity, biocompatibility and osteodifferentiation. Other coating techniques, such as hydrothermal synthesis [13], aerosol deposition [14], ion beam deposition [15] have also been explored for creating bioactive HA coatings on PEEK. Despite the recent advancement in PEEK coating technology, most of the above mentioned techniques require sophisticated equipment, elevated temperatures or long processing 
time. In addition, many coating deposition techniques are "line of sight" processes, which cannot be deployed for complex, 3D printed bone scaffolds with sophisticated internal structures. Electrophoretic deposition (EPD) technique is a facile, versatile and low-cost coating deposition process, which has been employed to deposit coatings of charged particles onto 3D printed metal scaffolds (e.g. titanium [16]). Various organic and/or inorganic materials such as PEEK/bioactive glass [17], polymethylmethacrylate [18], and polystyrene colloidal crystals [19] have also been electrophoretically coated onto stainless steels for potential biomedical applications. However, this technique relies on the high electrical conductivity of the substrates, hence its application towards insulating PEEK implants is hampered.

Graphene, a 2D nanomaterial with sp2 carbon hybridization, has the huge advantage of high electrical conductivity coupled with superior mechanical properties (modulus in the order of TPa [20]). Its unique multi-functionality also enabled its wide applications in biosensors [21], stem cell/tissue engineering [22, 23], drug/gene delivery [24], and bioimaging [25]. Incorporating $G$ into polymers can lead to nanocomposites with greatly enhanced electrical conductivity [26-29]. Such feature can facilitate the electrical stimulus to modulate and support the growth of bone cells and nerve cells [30], and has been exploited in regenerative medicine including cardiac and neural tissue engineering [31]. In addition, G possesses high photothermal conversion efficiency [32], which can be employed for cancer photothermal therapy and eradication of drug resistant bacteria [33,34]. Given the unique properties of G, it is hypothesized that addition of $G$ nanosheets into PEEK could lead to 
nanocomposites with desirable biomechanical properties and functionalities, which can be deployed for advanced orthopedic implants for bone repair as well as management of certain bone related diseases.

In this study, we designed and developed conductive PEEK/G nanocomposites with electrophoretically deposited bioactive, anti-bacterial STAC-HA coating (Scheme 1). The excellent electrical conductivity of the composite also allows for versatile coating deposition on complex $3 \mathrm{D}$ printed scaffold structures. The coated composites demonstrated remarkable properties including improved bulk mechanical properties, enhanced BMSCs proliferation and osteointegration, anti-bacterial properties as well as strong photothermal conversion effect under near infrared laser (NIR) irradiation. The material's unique photothermal property has also been demonstrated successfully for tumor inhibition and bacteria eradication. The ideal material combination and excellent features offered by our implant are desirable for bone regeneration as well as potential applications in osteosarcoma and osteomyelitis, which are often associated with large bone defects, diseases recurrence (due to residual cancer cells or bacteria), and post-surgery infection [32]. 

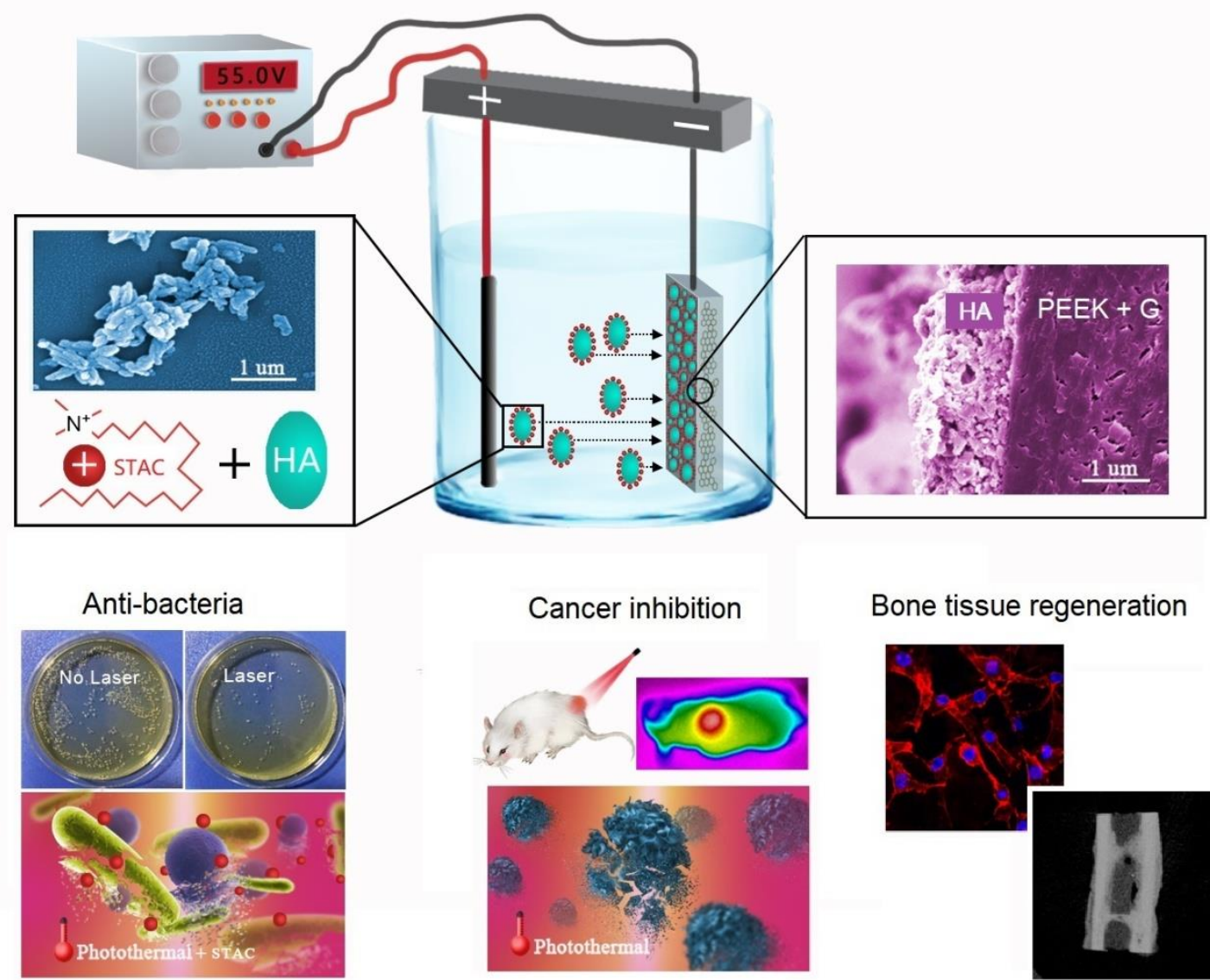

Bone tissue regeneration

Scheme 1. Schematic showing preparation of PEEK/G composite with EPD coating and its associated multi-functions.

\section{Results and discussion}

\section{Materials characterization}

Figure 1 shows that the addition of $G$ into PEEK has significantly enhanced the mechanical and electrical properties of P/G5 and P/G10. In particular, addition of 5\% $\mathrm{G}$ has led to over $60 \%$ increase in tensile modulus and nearly two-fold increase in compressive modulus as compared to that of pure PEEK (Figure 1a and 1b). Further increasing $\mathrm{G}$ to $10 \%$ has led to a slight drop in the composite mechanical properties, which is consistent with the reported literature [35]. The electrical conductivity of $\mathrm{P} / \mathrm{G} 5$ and P/G10 are $2 \times 10^{-3} \mathrm{~S} / \mathrm{cm}$ and $2.8 \times 10^{-3} \mathrm{~S} / \mathrm{cm}$, respectively (Figure 1c). This 
is in a similar range with semiconductor, and is twelve order of magnitude higher than that of the pure PEEK $\left(\sim 10^{-15} \mathrm{~S} / \mathrm{cm}\right)$. Such significant enhancement seen for electrical conductivity is consistent with our previous findings, where formation of electrical percolation network was achieved at $10 \mathrm{wt} \% \mathrm{G}$ concentration in a similar composite system deploying $G$ of the same size and aspect ratio [36]. To further reveal the chemical information of the composites, XPS analysis was carried out on a selected composite sample (P/G10). The characteristic peaks associated with oxygen containing groups are shown in Figure 1d, where $531.8 \mathrm{eV}$ is attributed to $\mathrm{O}=\mathrm{C}$ and $533.2 \mathrm{eV}$ to O-C. Other characteristic peaks at $284.6 \mathrm{eV}, 286.2 \mathrm{eV}$ and $288.3 \mathrm{eV}$ have been assigned to $\mathrm{C}-\mathrm{C} / \mathrm{C}-\mathrm{H}$ bond, $\mathrm{C}-\mathrm{O}$ bond and, $\mathrm{C}=\mathrm{O}$ bond, respectively. It is worth noting that the bond at $291.4 \mathrm{eV}$ has been ascribed to $\pi-\pi^{*}$ conjugation between $\mathrm{G}$ and PEEK (Figure 1e) [9]. The $\pi-\pi^{*}$ conjugation provides an alternative electron migration pathway, which could also partly contribute to the enhanced electrical conductivity of the composites, in addition to the $\mathrm{G}$ electrical percolation network, see schematic in Figure 1f. 

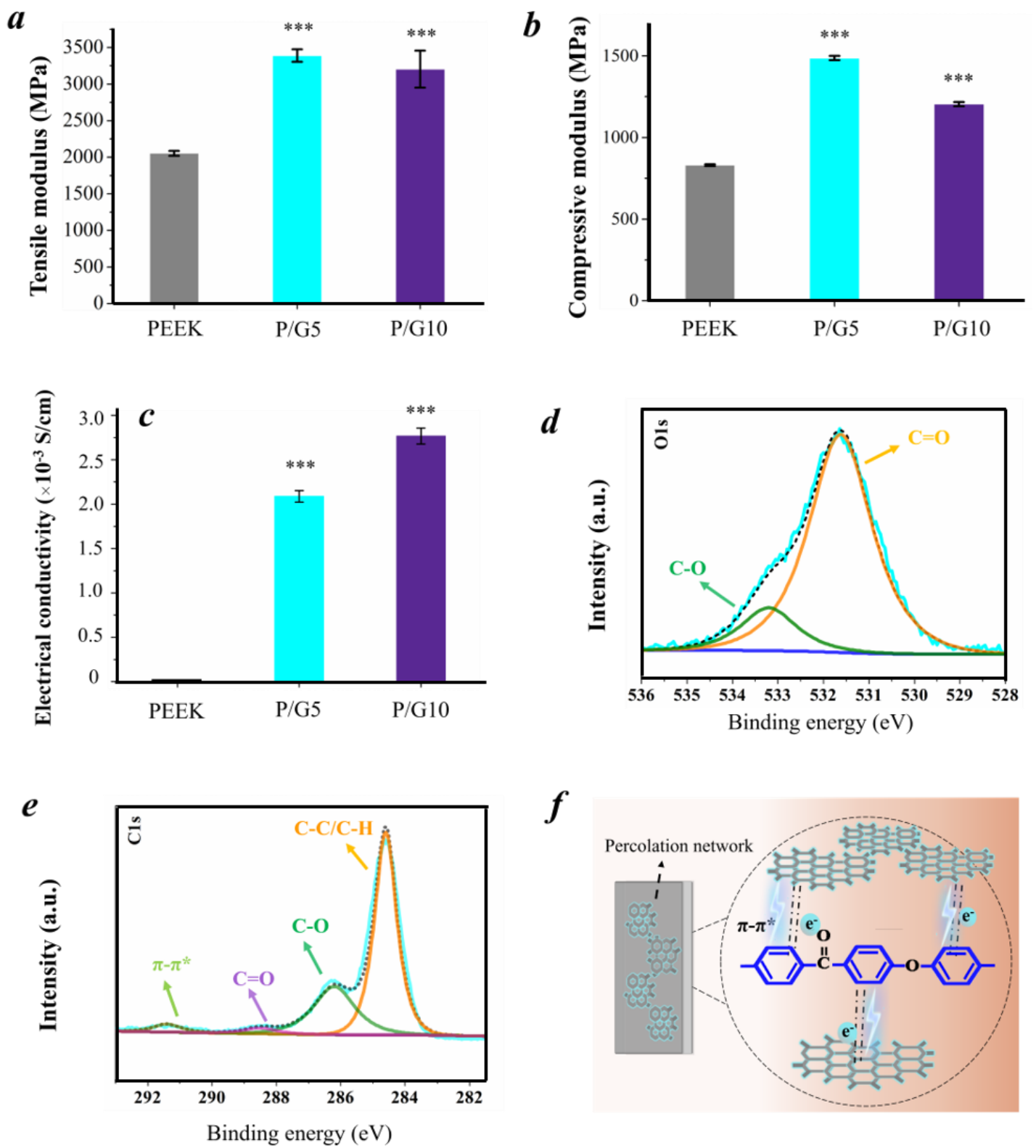

Figure 1. The tensile moduli (a) compressive moduli (b) and electrical conductivity (c) of samples, $* * *$ p $<$ $0.001, * * p<0.01, * \mathrm{p}<0.05$ vs. pure PEEK; XPS curves showing deconvolution of O1s (d) and C1s (e) peaks of P/G10; (f) schematic showing mechanisms of enhanced electrical conductivity in PEEK/G composites.

Given P/G10 offers the best electrical conductivity as well as sufficiently improved mechanical properties, it is selected for subsequent EPD process as well as further testing/analysis. Figure $\mathbf{2 a}$ and $\mathbf{2 b}$ show that the plasma activated P/G10 surface has abundant oxygen rich functional groups as compared to the pure PEEK. Specifically, the characteristic peaks at $284.6 \mathrm{eV}, 286.2 \mathrm{eV}$ and $288.1 \mathrm{eV}$ correspond 
to $\mathrm{C}-\mathrm{C} / \mathrm{C}-\mathrm{H}$ bond, $\mathrm{C}-\mathrm{O}$ bond and $-\mathrm{COO}$ bond respectively $[37,38]$. Oxygen containing groups are located at $533.5 \mathrm{eV}(-\mathrm{COOH}), 532.8 \mathrm{eV}(\mathrm{C}-\mathrm{O}-\mathrm{C} / \mathrm{C}-\mathrm{OH})$ and $531.8 \mathrm{eV}(\mathrm{C}=\mathrm{O})$, respectively $[39,40]$. The rich surface $-\mathrm{COOH}$ may become deprotonated and negatively charged when immersed in aqueous solution, hence facilitating the subsequent deposition of positively charged STAC-HA particles. Figure 2c shows the Zeta potential of STAC-HA suspension at different HA:STAC ratio. At HA:STAC ratio of 5:2, the suspension yielded a strong positive Zeta potential $(>30 \mathrm{mV})$ and exhibited the Faraday-Tyndall effect (Figure 2c inset) even after $24 \mathrm{~h}$, which confirmed the stable dispersion of nanoscale STAC-HA particles.
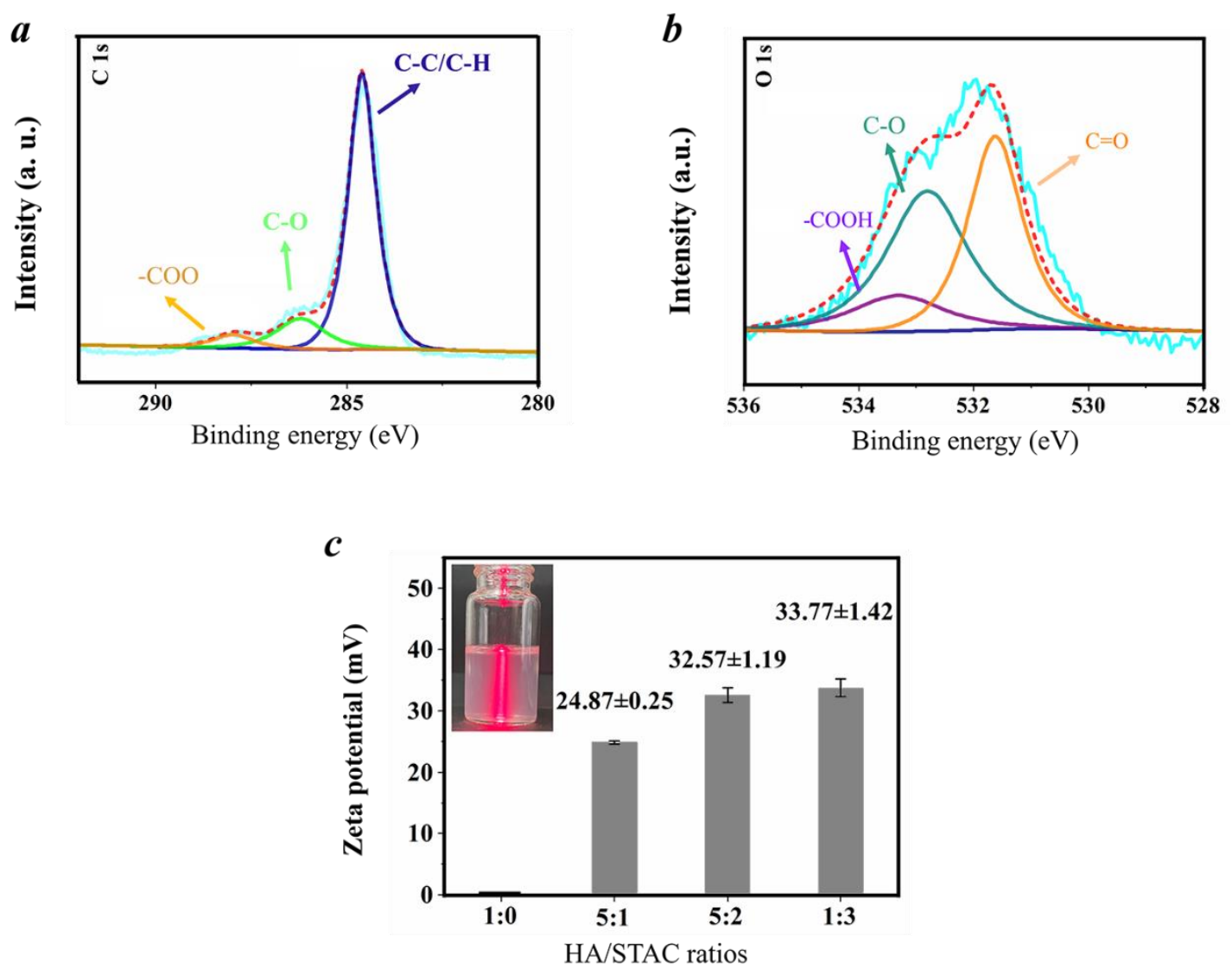

Figure 2. XPS curves showing deconvolution of C1s (a) and O1s (b) peaks of plasma activated P/G10; (c) Zeta potential of STAC-HA suspensions with different of HA/STAC ratios, inset: Faraday-Tyndall effect of a typical STAC-HA suspension. 
Figure 3a-c shows the morphology of pristine HA, dispersed STAC-HA particles as well as EPD coating on P/G10 substrate. The EPD coating is approximately $18 \mu \mathrm{m}$ thick (Figure 3d) and features stacked clumps made of needle-like HA crystals with similar crystal spacing to that of the pristine HA. Corresponding EDS analysis (Figure S2) shows that the coating is composed of $\mathrm{Ca}$ and $\mathrm{P}$ with a $\mathrm{Ca} / \mathrm{P}$ ratio of 1.69 , similar to the $\mathrm{Ca} / \mathrm{P}$ ratio in stoichiometric $\mathrm{HA}$ [41]. It is worth mentioning that the STAC surface modification did not affect the morphology of the rod-like HA crystals $[42,43]$. However, the significant change of HA morphology within the EPD coating implies the strong effect of electric field on the morphology and orientation of HA crystals. The scratch test showed that the bonding strength of HA coating and P/G10 substrate is approximately $315 \mathrm{mN}$ (Figure S3). This is comparable to the bonding strength reported previously for HA coatings on Ti6Al4V alloy [44]. 


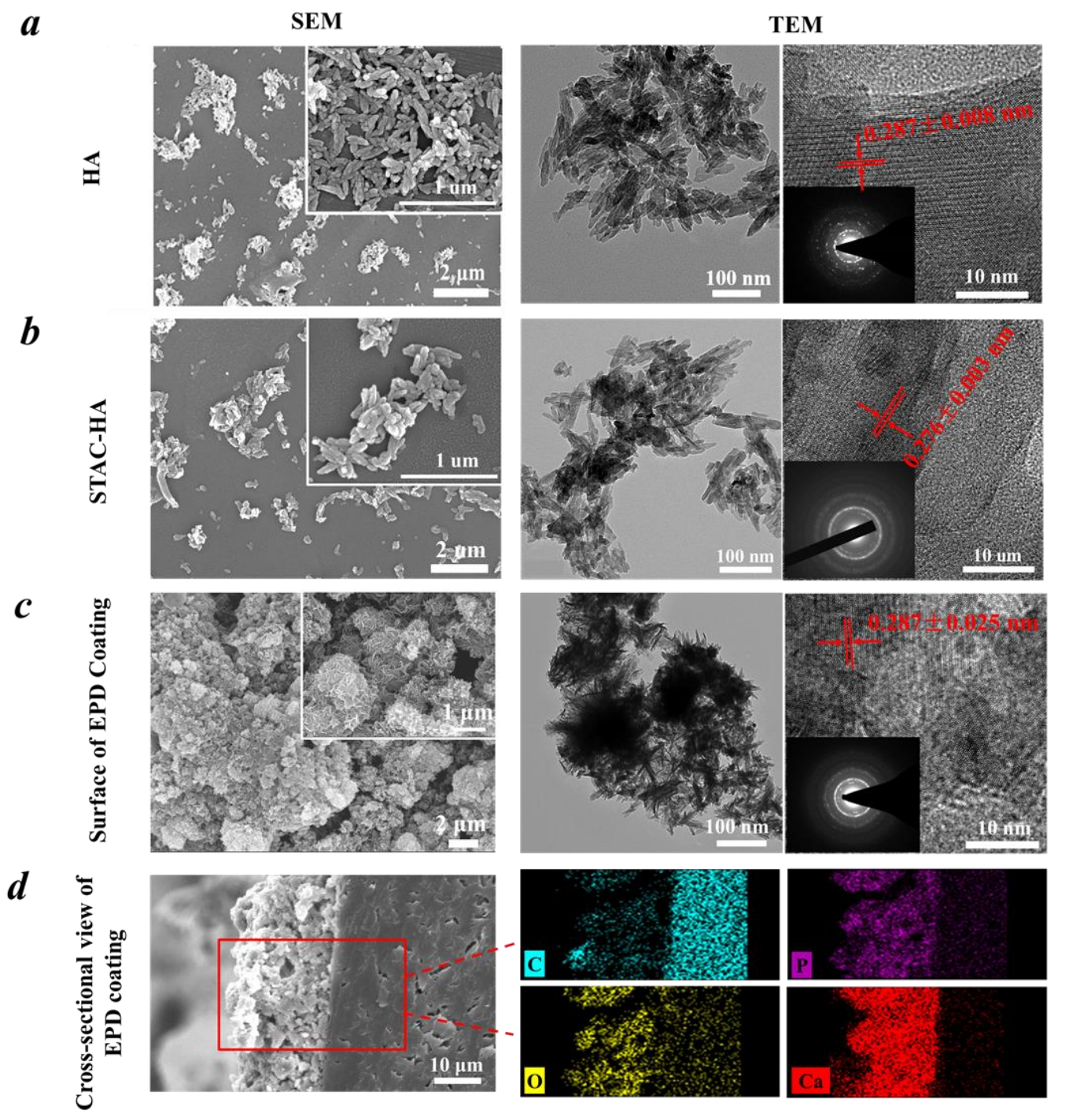

Figure 3. Top view SEM and TEM images of HA (a), STAC-HA (b) and EPD coating (c); (d) cross-sectional SEM image and EDS analysis of the EPD coating.

XPS analysis in Figure 4a shows that the EPD STAC-HA coating features characteristic peak of $\mathrm{N} 1 \mathrm{~s}$ at $399.8 \mathrm{eV}(\mathrm{C}-\mathrm{N})$ and $402.4 \mathrm{eV}\left(\mathrm{C}-\mathrm{N}^{+}\right)[45,46]$, which confirms the STAC modified HA has been successfully deposited onto the plasma-treated PEEK substrate. The hydrophobicity the samples was analyzed and the water contact angle of PEEK and P/G10 were $78.74^{\circ}$ and $73.12^{\circ}$, respectively. Plasma treatment of P/G10 has led to significantly enhanced surface hydrophilicity (contact angle $\sim 0^{\circ}$ ). However, the plasma treated surface passivated rapidly when 
exposed in air and yielded a contact angle of $\sim 50.70^{\circ}$ after $15 \mathrm{~min}$. In contrast, a near $0^{\circ}$ contact angle can be maintained on the STAC-HA coating over time, indicating the coating's highly stable hydrophilic nature (Figure $\mathbf{4 b}$ ).

As can be seen from Figure 4c, HA and STAC-HA exhibit identical XRD patterns. In contrast, the EPD coating has weakened diffraction peaks at (002), (211), (301), implying the decreased crystallinity; but the much stronger peak at $2 \theta=28.9^{\circ}$ which signifies the preferred orientation in (210) direction. During the coating preparation process, the positively charged STAC-HA will initially adsorb onto the $-\mathrm{COO}^{-}$groups on PEEK surface through strong electrostatic interaction $[47,48]$. The subsequent application of the electric field would have an influence on the dipole moment vectors of STAC-HA crystals, leading to their preferential orientation along the field direction [49]. The curves in Figure 4d shows that greater amount of $\mathrm{Ca}^{2+}$ ions were released from the EPD coating comparing to that from the pristine HA. This may be because the EPD process has decreased the crystallinity of HA crystals and increased its specific surface area, which facilitates the release of $\mathrm{Ca}^{2+}$.

It is also worth mentioning that the material preparation technique reported here could be generalized to a range of coating materials and substrate structures. For instance, coating consisting of titanium dioxide $\left(\mathrm{n}-\mathrm{TiO}_{2}\right)$ nanoparticles has been successfully deposited onto the PEEK/G via our EPD technique (Figure S4) and the STAC-HA coating can also be successfully deposited onto complex 3D printed P/G10 scaffold (both surface and interior) as shown in Figure S5. 

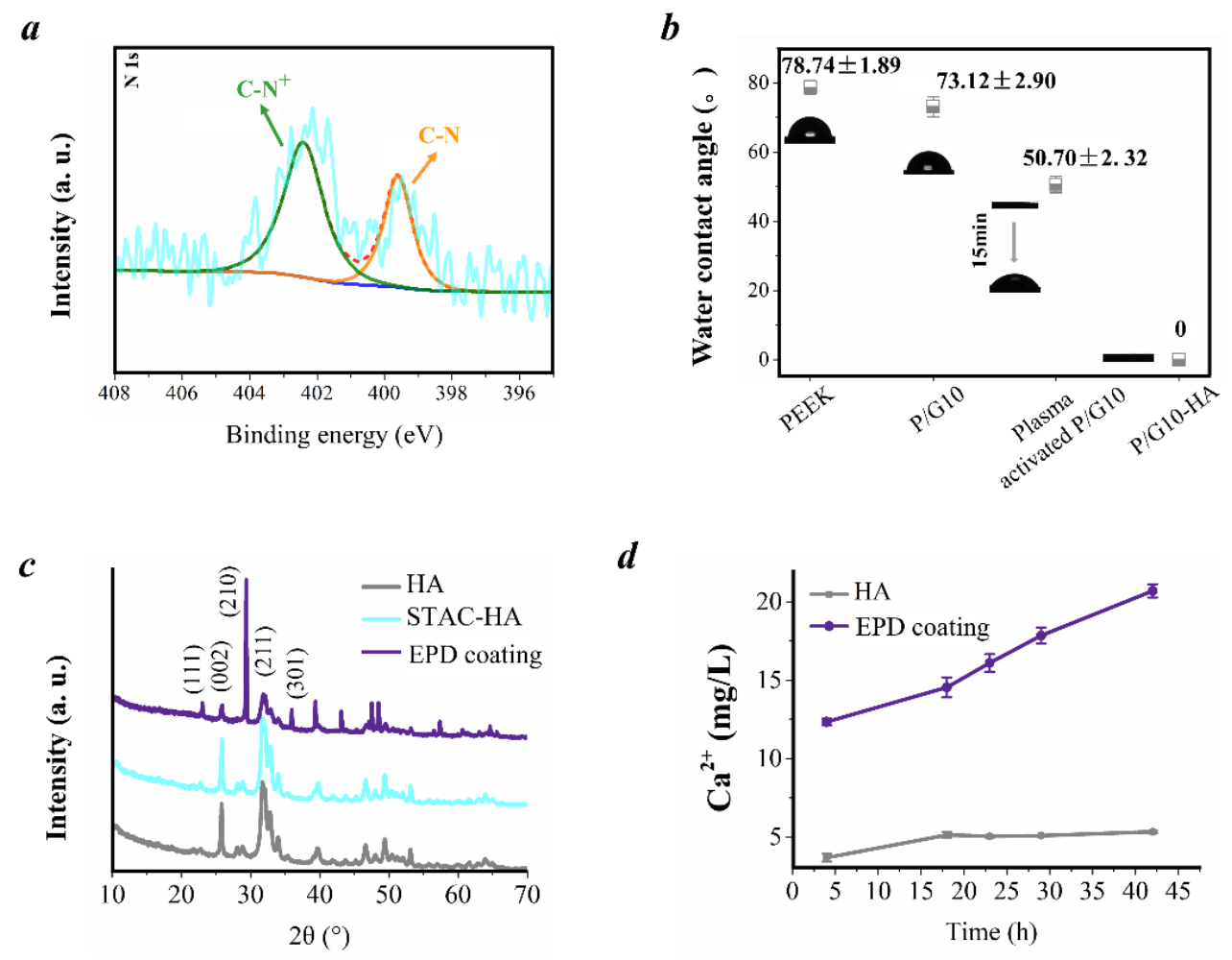

Figure 4. (a) XPS curves showing deconvolution of N1s within the EPD coating; (b)Water contact angle on different sample surface; (c) XRD spectra; (d) The release curves of $\mathrm{Ca}^{2+}$ from pristine $\mathrm{HA}$ and the EPD coating in physiological saline.

\section{Functional characterization}

\section{Photothermal conversion effect}

Figure 5a-c shows the NIR thermal images of PEEK, P/G10 and P/G10-HA under laser irradiation $\left(808 \mathrm{~nm}, 0.31 \mathrm{~W} / \mathrm{cm}^{2}\right)$ when immersed in PBS solution (temperature data recorded in air can be found in Figure S6). Pure PEEK showed no distinct temperature change in PBS throughout the testing period, whereas the average temperature of P/G10 and P/G10-HA increased significantly over time. Specifically, under laser power density of $0.43 \mathrm{~W} / \mathrm{cm}^{2}$, the temperature of P/G10 increased from 25 to $45^{\circ} \mathrm{C}$ within $100 \mathrm{~s}$ (at the point of irradiation). The strong photothermal conversion effect can be attributed to the electron transition of $G$ from its ground state to the 
excited state, followed by energy relaxation through nonradiative decay. This process has led to an increase in the kinetic energy to drastically overheat the local environment around the light-absorbing species [50]. For P/G10-HA, despite the temperature rise was slightly delayed due to the barrier effect of coating, the sample was heated up to $40 \sim 45^{\circ} \mathrm{C}$ within $150 \mathrm{~s}$, reaching the therapeutic window required for cancer phototherapy. The data suggests that it is possible to adjust the degree of photothermal conversion of the composite samples by manipulating the NIR power density and the irradiation time. Figure 5d shows temperature profile of P/G10-HA under heating/cooling cycles. There was no significant attenuation during the repeated cycles, suggesting the sample is thermally stable and can be used repetitively for photothermal conversion applications.
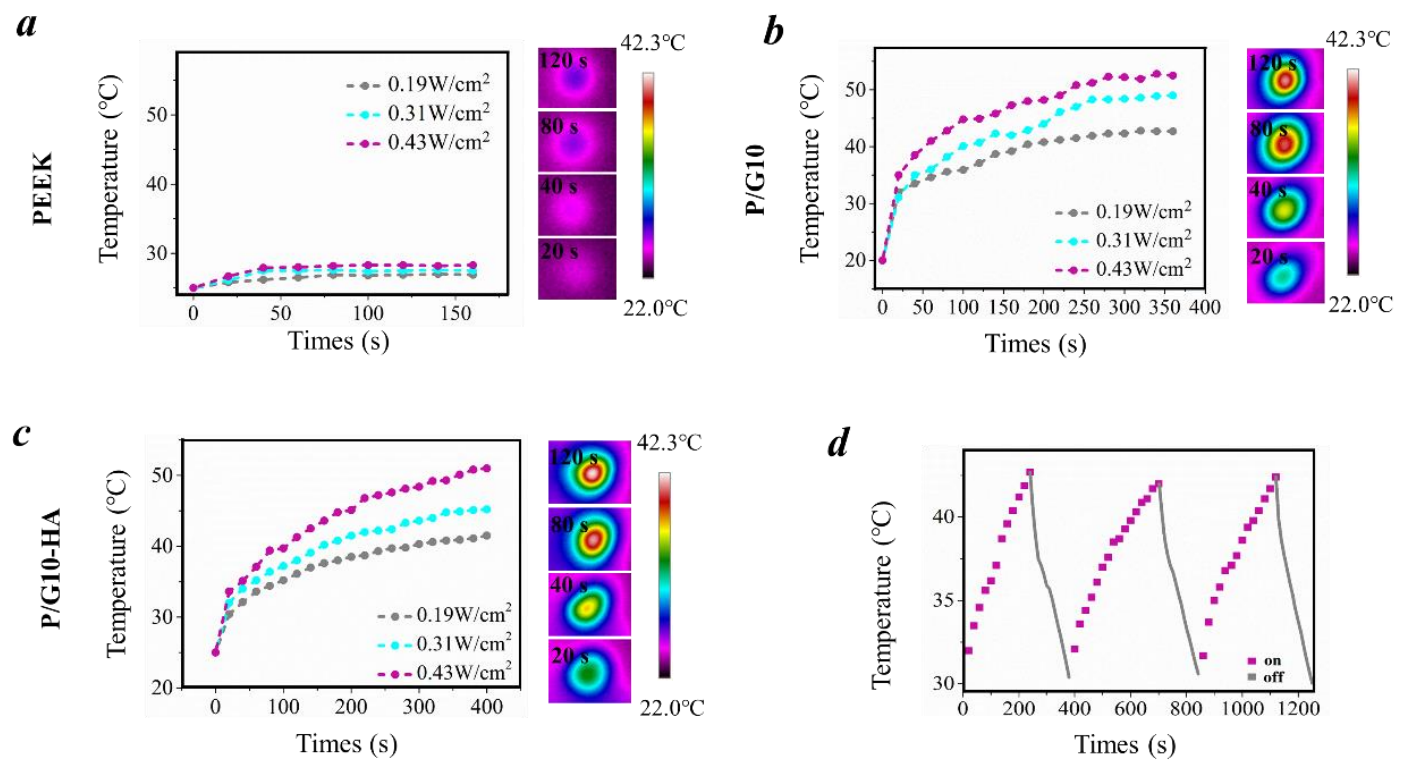

Figure 5. Temperature variation of PEEK (a), P/G10 (b) and P/G10-HA (c) under laser irradiation with different laser power densities $\left(0.19 \mathrm{~W} / \mathrm{cm}^{2}, 0.31 \mathrm{~W} / \mathrm{cm}^{2}, 0.43 \mathrm{~W} / \mathrm{cm}^{2}\right)$ in PBS solution and the corresponding NIR thermal images (laser power density $=0.19 \mathrm{~W} / \mathrm{cm}^{2}$ ); (d) Temperature of P/G10-HA under ON/OFF cycles (laser power density $=0.19 \mathrm{~W} / \mathrm{cm}^{2}$ ) 


\section{Antibacterial property}

Osteomyelitis and/or postoperative deep bacterial infection after bone surgeries present significant health challenges [51]. To address this issue, we incorporated anti-bacterial drug STAC into the HA coating to serve as anti-bacterial agents for broad spectrum gram-negative and gram-positive bacteria. The test for antibacterial property demonstrated the bacteriostatic rate (BR) of P/G10-HA against S. aureus and E. coli were $99.99 \%$ and $56.82 \%$, respectively, see Figure 6a, Figure S7 and S8. One episode of laser irradiation $\left(0.31 \mathrm{~W} / \mathrm{cm}^{2}, 10 \mathrm{~min}\right)$ can lead to significantly enhanced BR against E. coli $(91.00 \%$, see Figure $\mathbf{6 b})$, indicating the potential of achieving near total eradication of both bacterial strains via synergistic photothermal and antibiotic bacteria killing. It is also worth mentioning that laser irradiation on our composite samples has also led to the generation of single oxygen, see Figure 6c. The $\pi-\pi^{*}$ conjugation between $\mathrm{G}$ and PEEK acts as fast electron migration pathway for enhanced separation of electron-hole pairs [52]. The photo-activation of graphene may generate reactive oxygen species by electron/energy transfer to the surrounding free oxygen, and the resulting reactive species may work synergistically with STAC and the light induced heating in killing of bacteria [53-55]. 
a

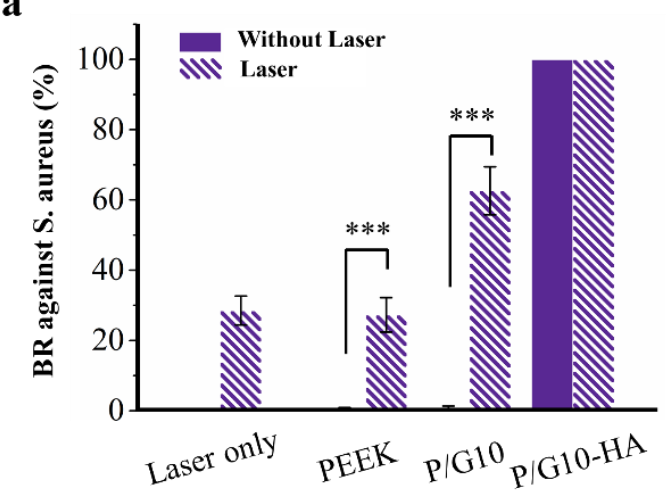

b

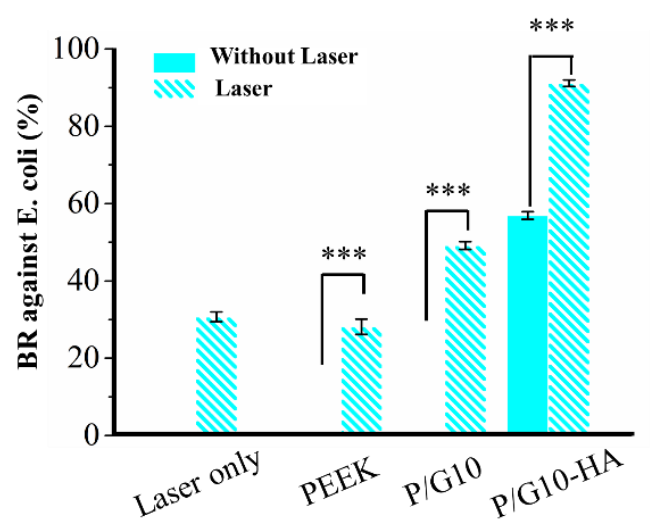

c
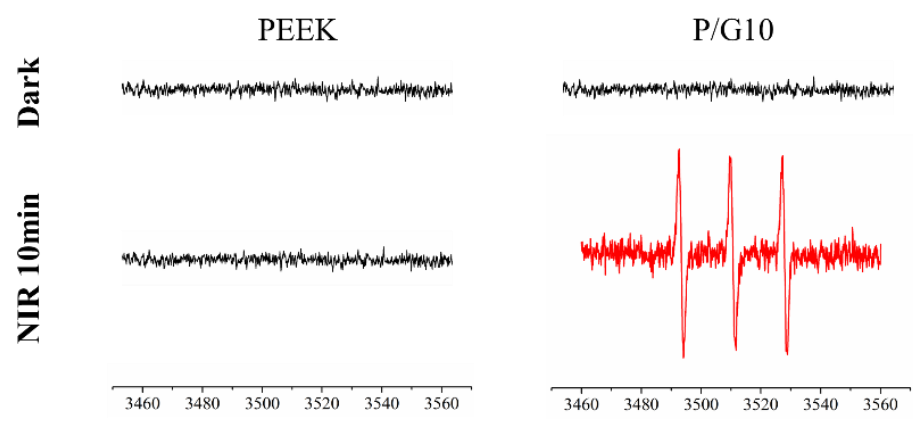

P/G10-HA

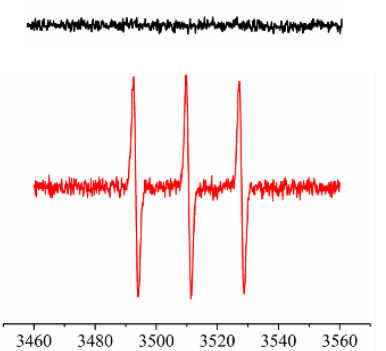

Figure 6. Bacteriostatic rate against $S$. aureus (a) and $E$. coli (b) of $\mathrm{P}, \mathrm{P} / \mathrm{G} 10$ and $\mathrm{P} / \mathrm{G} 10-\mathrm{HA}$ with and without laser irradiation, $* * * p<0.001, * * \mathbf{p}<0.01, * \mathbf{p}<0.05$ vs. without laser; (c) ESR spectra of single oxygen generation on PEEK, P/G10 and P/G10-HA

In vitro and in vivo studies

Cytocompatibility and bone regeneration

The BMSCs morphologies were recorded using SEM, CLSM and live-dead stain technique (Figure 7a and Figure S9). Cells displayed a good growth and spreading condition on all samples. The live-dead cell images suggested that the active cells (green fluorescence) predominated. Moreover, MTT assay (Figure 7b) revealed that BMSCs proliferated well on PEEK, P/G10 and P/G10-HA over the testing period of 7 d. Particularly, the absorbance of P/G10-HA was greater than that of PEEK and P/G10. This suggests the presence of EPD coating on PEEK/G composite favors the growth of BMSCs, which can be associated with the greater $\mathrm{Ca}^{2+}$ release [56] as discussed in 
Figure 4d. The cross-section CT scan in Figure 7c confirms more bone tissue surrounds the P/G10-HA as compared with $\mathrm{P}$ and P/G10. Figure 7d provides the quantitative analysis of bone histomorphometry indices (BV/TV). It can be seen that the BV/TV of P/G10-HA was much greater than that of the control. This suggests that the EPD coating has significantly boosted the proliferation and osteoblastic potential of cells in vitro and hence can promote new bone tissue formation.
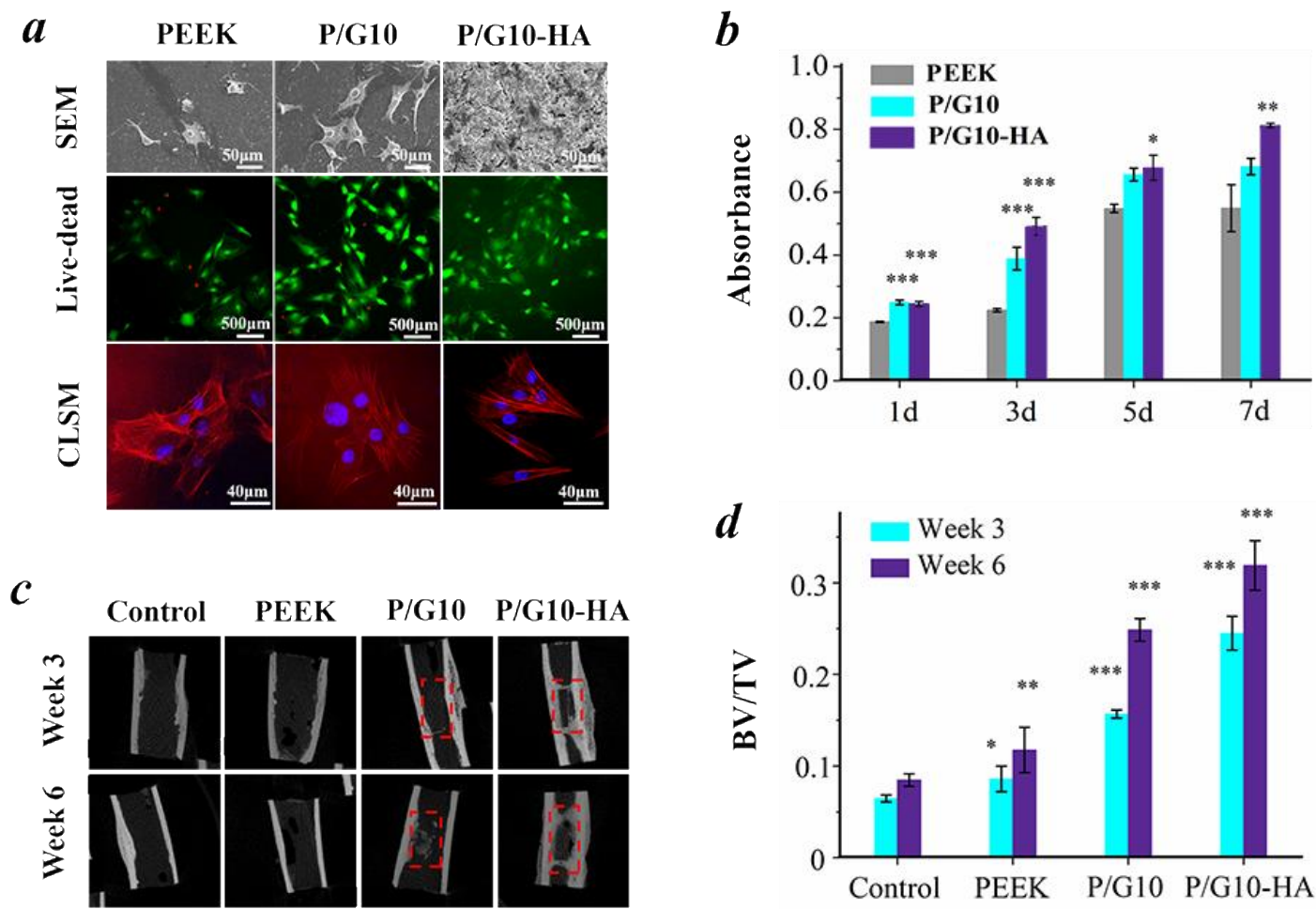

Figure 7. (a) SEM, Live-dead and CLSM of BMSCs cultured with different samples for $1 \mathrm{~d}$; (b) MTT of BMSCs on PEEK, P/G10 and P/G10-HA for $1 \mathrm{~d}, 3 \mathrm{~d}, 5 \mathrm{~d}$ and $7 \mathrm{~d}$ respectively, $* * * p<0.001, * * p<0.01, * p<0.05$ vs PEEK; (c) the cross-section images of CT scan; (d) BV/TV of different samples, $* * * p<0.001, * * p<0.01$, $* p<0.05$ vs control.

\section{Tumor inhibition}

In this study, MG63 cells were selected as the model cells to study the photothermal inhibition effect of our composites towards osteosarcoma cells. 
Qualitative analysis of the cell adhesion was first carried out using SEM, live-dead and CLSM images. It can be seen from Figure 8a that MG63 cells displayed similar morphology and spreading behaviour before laser irradiation. All cells displayed strong pseudopodia attachment on the surfaces in all directions on different sample. Upon laser irradiation $\left(0.31 \mathrm{~W} / \mathrm{cm}^{2}\right.$ for $\left.10 \mathrm{~min}\right)$, cells on $\mathrm{P} / \mathrm{G} 10$ and $\mathrm{P} / \mathrm{G} 10-\mathrm{HA}$ exhibited typical rounded morphology, indicating the normal growth of MG63 has been inhibited. The results can be further coroborated by CLSM and live-dead staining images (Figure S10 and S11), where MG63 cells exhibited greater death rate (red flurescence) on P/G10 and P/G10-HA after laser irradiation.

To quantify the inhibition effect of laser irradiation towards the proliferation of MG63, MTT analysis has been carried out (Figure 8b). It can be seen that the cell absorbance data for pure PEEK displays no senstivity towards laser irradation. In contrast,the absorbance of MG63 cultured on P/G10 and P/G10-HA showed sharp decrease with inceased laser power density and irradiation time. Most significant drop in cell absortion (from 0.50 to below 0.2 ) was seen for samples irradiated by 0.43 $\mathrm{W} / \mathrm{cm}^{2}$ laser for $15 \mathrm{~min}$. 
$a$
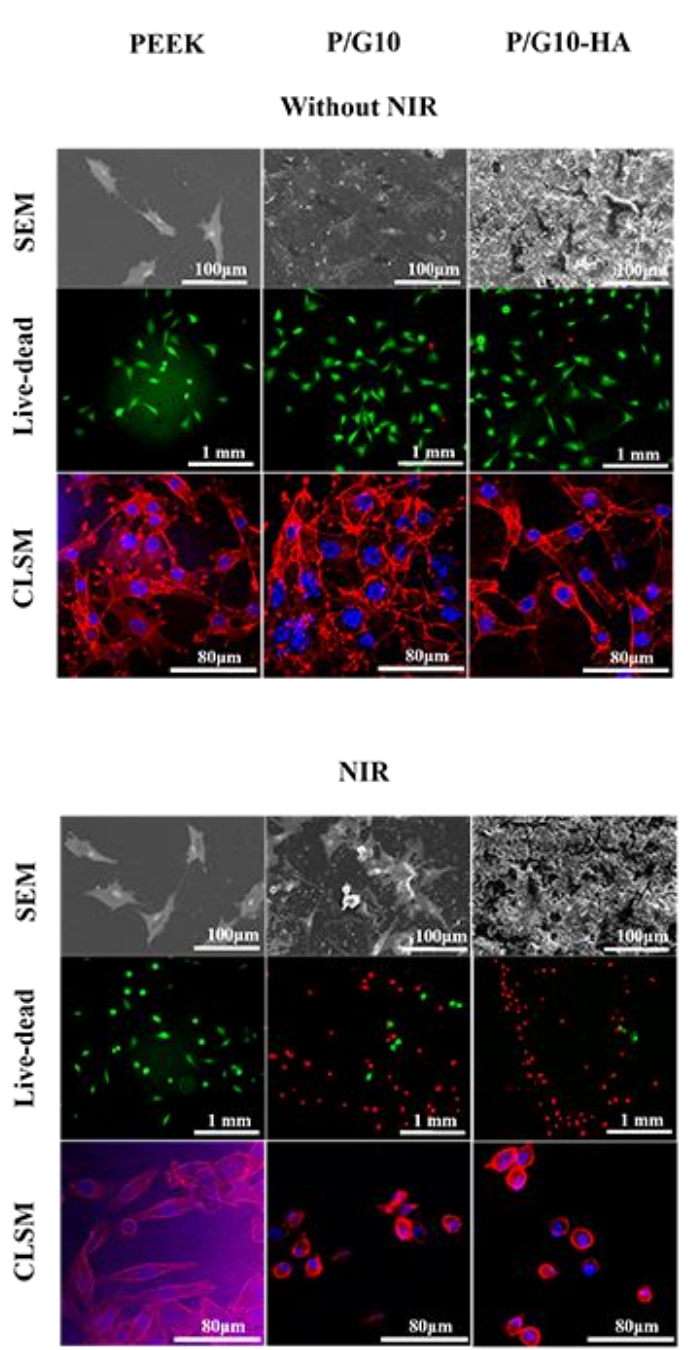

b
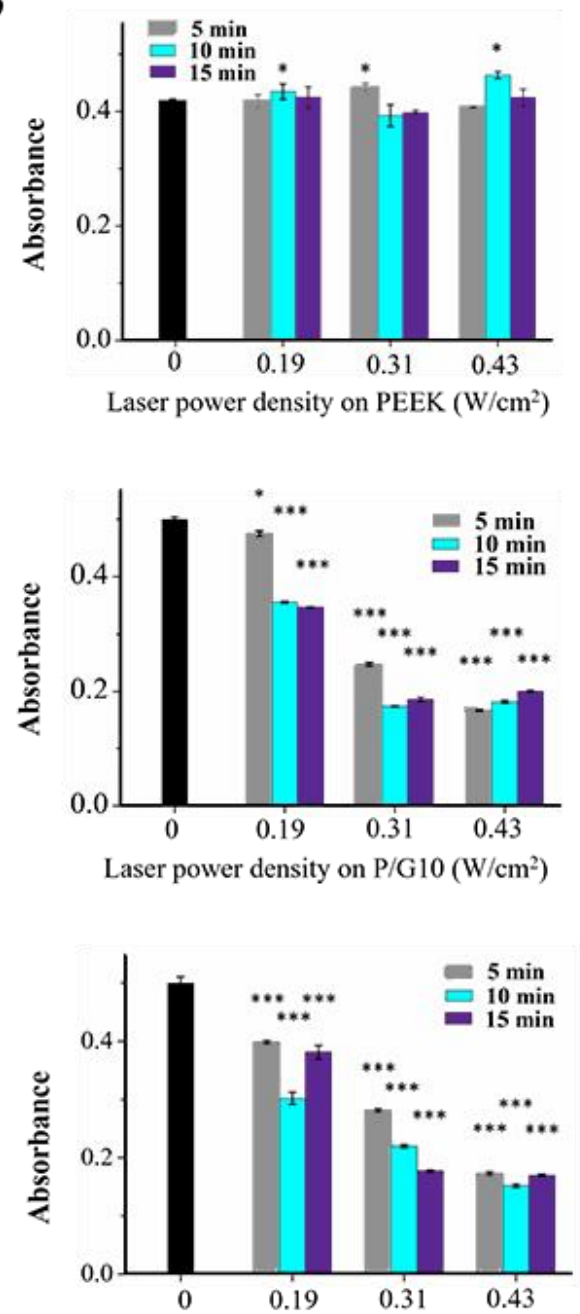

Laser power density on $\mathrm{P} / \mathrm{G} 10-\mathrm{HA}\left(\mathrm{W} / \mathrm{cm}^{2}\right)$

Figure 8. (a) SEM, live-dead images and CLSM images of MG63 with and without laser irradiation respectively; (b) MTT of PEEK, P/G10 and P/G10-HA with different power density and irradiated time respectively, $* * * p<0.001, * * p<0.01, * p<0.05$ vs the sample without laser irradiation.

The effectiveness of cancer photothermal therapy enabled by our composite samples was also verified in vivo by conducting daily laser treatment on nude mouse for a course of 14 days. P/G10 and P/G10-HA which present strong photothermal conversion effect (Figure 9a) have effectively inhibited the tumor growth, as is shown in Figure 9b. Figure 9c shows that without daily laser treatment, the tumor volume $\left(\mathrm{V} / \mathrm{V}_{0}\right)$ rose sharply over time (up to 13 -fold volume increase by day 14$)$. The 
weight of nude mouse maintained stable over the treatment period (Figure S12).

Further H\&E staining of the tumor tissues confirmed the nucleus of tumor cells under laser irradiation demonstrated much stronger degrees of tumor tissue contraction in P/G10 and P/G10-HA due to the laser heating (Figure 9d).

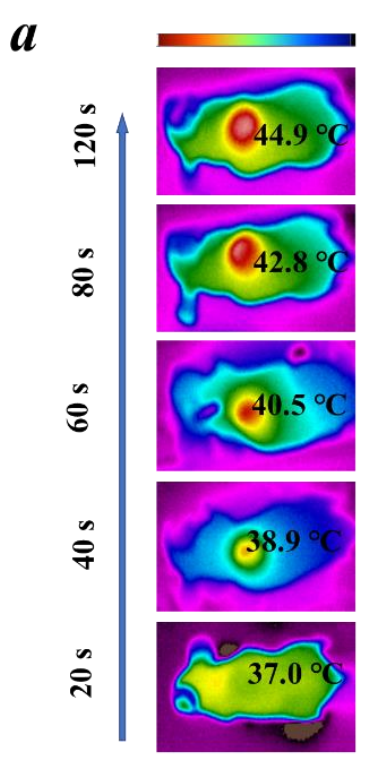

$c$

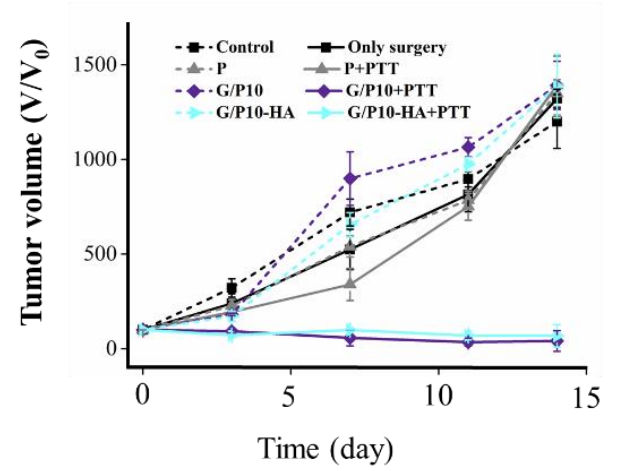

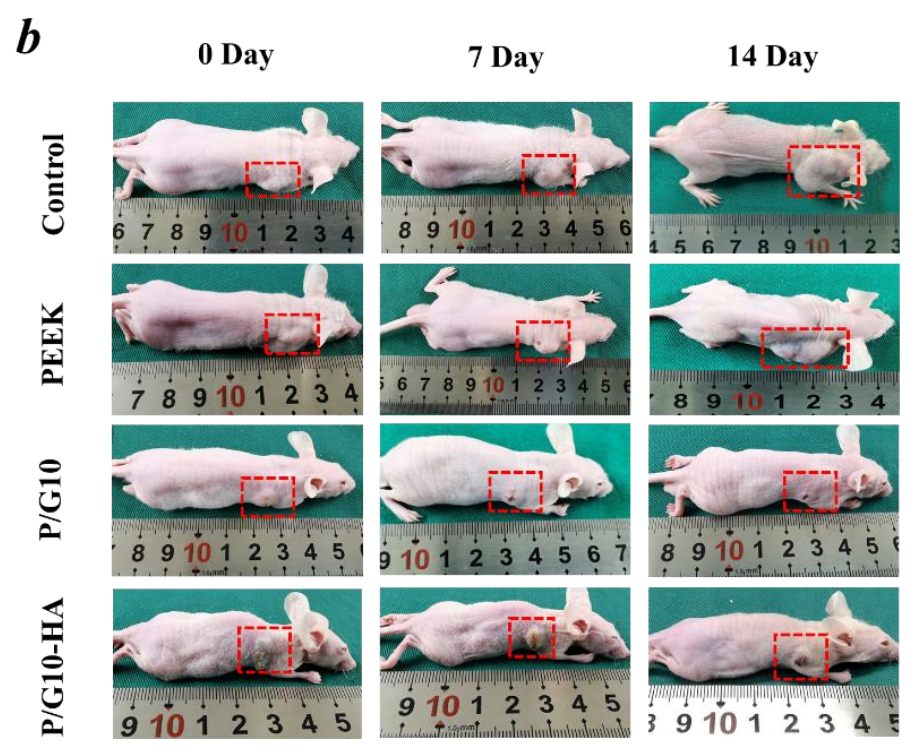

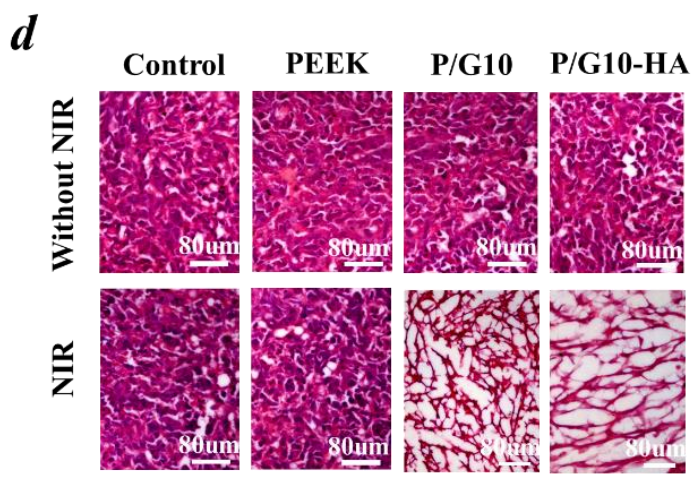

Figure 9. (a) NIR image showing photothermal effect of P/G10-HA in vivo; (b) photograph showing tumor bearing mice in different treatment groups; (c) tumor volume in each treatment group as a function of time; (d) H\&E staining of extracted tumor tissues from different treatment groups on Day 7.

\section{Conclusion}

In this study, we created a multi-functional implant material consisting of electrically conductive PEEK/graphene nanocomposite with electrophoretically 
deposited STAC-HA coating. The bioactive coating can significantly enhance bone tissue regeneration while offering anti-bacterial effects towards both $E$. coli and $S$. aureus bacterial strains. Results also show that our composite implants can enable effective photothermal conversion effect, the temperature profile of which can be manipulated by turning the NIR laser power density and irradiation time. The strong photothermal conversion effect of the implant, when combined with anti-bacterial drug laden coating, has led to enhanced bacteriostatic rate (91\% against E. coli and 99.99\% against S. aureus). Additionally, the photothermal properties of our implant has also been demonstrated successfully for tumor inhibition studies in vivo. The material preparation technique proposed in this study can be generalized for creation of different functional surfaces (such as $\mathrm{TiO}_{2}$ coating), and for implants with highly complex architectures (e.g., 3D printed scaffold, etc). We believe that such unique material design may provide effective solution towards bone repair as well as enabling multi-modal therapeutics towards the management of challenging bone diseases such as osteosarcoma and osteomyelitis.

\section{Materials and methods}

Materials. Highly osteoconductive hydroxyapatite (HA) was synthesized by hydrothermal method according to established processes $[57,58]$. Positively charged stearyltrimethylammonium chloride (STAC) (Aladdin, China) was attached to HA particles through physisorption and a stable stock solution of STAC modified HA suspension (STAC-HA) was used for subsequent coating deposition. 
Polyetheretherketone (PEEK) powder with the particle size of $500 \mu \mathrm{m}$ (Jilin Joinature Polymer Co., Ltd., China) was dried at $80^{\circ} \mathrm{C}$ in an oven for $12 \mathrm{~h}$ before use. Graphene (G) nanosheets ( $~ 5$ layers, maximum radial size $2 \sim 4 \mu \mathrm{m}$, average aspect ratio 4500$)$ were provided by the Sixth Element (Changzhou) Materials Technology Co., Ltd. China. PEEK powder was mixed with $5 \mathrm{wt} \%$ and $10 \mathrm{wt} \% \mathrm{G}$ respectively and the mixtures were dispersed in ethanol by sonication for 30 min at $25{ }^{\circ} \mathrm{C}$. After filtration and drying, the mixture powders were moulded into $10 \mathrm{~cm} \times 10 \mathrm{~cm} \times 1 \mathrm{~mm}$ sheets by hot pressing with the temperature of $380{ }^{\circ} \mathrm{C}$ and pressure of $2 \mathrm{MPa}$ (YJAC, Chengdu Hangfa Hydraulic Engineering Co., Ltd, China). The resulting composites were named as P/G5 and P/G10, respectively. The composites were then exposed to low-temperature $\mathrm{O}_{2} / \mathrm{Ar}$ plasma system (Diener Electronic. Germany) under a pressure of $0.3 \mathrm{mbar}$, a gas flow of $8.3 \mathrm{sccm}$ for $10 \mathrm{~min}$ for surface activation.

Electrophoretic deposition (EPD). Plasma treated P/G10 was selected as a typical sample for electrophoretic deposition of STAC-HA coating. A carbon rod (anode) and P/G10 (cathode) were immersed in the STAC-HA suspension in the electrochemical cell. EPD was carried out for 90 min under an applied DC voltage of $55 \mathrm{~V}$.

Characterization. Tensile and compressive tests were performed using universal mechanical testing machine (MTS, model E45, USA), and the speed was set as 1 $\mathrm{mm} / \mathrm{min}$. The electrical conductivity of all samples was measured by four-point probes method (RTS-8, Tianjin Nuleixinda Technology Co., Ltd., China). X-ray photoelectron spectroscopy analysis (XPS, XSAM800, Kratos, England) was 
performed to confirm the state of material surface chemistry. Zeta potential (Zetasizer Nano ZS, Malvern, England) was used to determine the surface charge of the STAC-HA particles. The surface wettability of samples was recorded by contact angle measuring instrument (JY-82A, Chengde Dingsheng Co. LTD, China). The bonding strength between the substrate and the HA coating was measured by nanoscratch tester (UNHT, Anton Paar, Austria). Scanning electron microscope (JSM-7500F, JEOL, Japan), EDS (JSM-7500F, JEOL, Japan) and transmission electron microscope (TEM, Tecnai G2 F20 S-TWIN, FEI, America) were used to analyze the morphology, elements and diffraction pattern of HA, STAC-HA particles and EPD coating. X-ray diffraction (XRD, EMPYREAN, PANalytical B.V., Holland) was carried out to confirm the crystal orientation of $\mathrm{HA} . \mathrm{Ca}^{2+}$ ions dissolution from the coating was analyzed by ICP-OES (AXIS Ultra DLD, Kratos, UK) at different time intervals (4 h, $17 \mathrm{~h}, 23 \mathrm{~h}, 29 \mathrm{~h}$ and $42 \mathrm{~h}$ ) following established method [59].

The photothermal conversion effect of all samples was tested under immersed condition in phosphate buffered solution (PBS). A $808 \mathrm{~nm}$ NIR laser was used at different laser power densities $\left(0.19 \mathrm{~W} / \mathrm{cm}^{2}, 0.31 \mathrm{~W} / \mathrm{cm}^{2}, 0.43 \mathrm{~W} / \mathrm{cm}^{2}\right)$ and the sample's temperature change was monitored in real time using an infrared thermal imaging system (TiS20+, Fluke, USA). The temperature data were analyzed using FLUKE software.

Antibacterial testing. Gram-negative Escherichia coli (E. coli, ATCC25922) and gram-positive Staphylococcus aureus (S. aureus, ATCC25923) were used to evaluate the antibacterial properties of PEEK, P/G10 and P/G10-HA following 
standard protocols $[60,61]$. Specifically, ten-fold dilution method was used to quantitatively measure the bacteriostasis rate (BR) which is defined by Eq. (1) [62]. Briefly, bacterial strain was incubated in the culture medium for $24 \mathrm{~h}$ and the subculture from the second passage was used as the pre-made bacterial fluid. $100 \mu \mathrm{L}$ pre-made bacterial fluid was dropped onto samples and incubated for $2 \mathrm{~h}$ followed by addition of $2 \mathrm{~mL}$ physiological saline. The mixture of bacterial fluid and physiological saline extracted from each sample was subsequently diluted $10^{4}$ times. Finally, $100 \mu \mathrm{L}$ of each diluted fluid sample was inoculated on nutrient Luria-Bertani agar plate. The number of bacterial colonies up to 30 300 CFU (colony-forming unit) was counted.

$$
\mathrm{BR}(\%)=\frac{\mathrm{n}_{0}-n}{\mathrm{n}_{0}} \times 100 \%
$$

Where $n_{0}$ is the number of colonies on the control plate, and $n$ is the number of colonies on the plates of the experimental group.

To investigate the effect of laser irradiation on the implant antibacterial performance, a separate set of samples were irradiated by $808 \mathrm{~nm}$ NIR laser $(0-2$ W/cm², Richeng Science and Technology Development Co. LTD, Shanxi, China) for 10 min before the ten-fold dilution method was applied. Electron spin resonance (ESR, Bruker A300, Germany) was used to measure the free radicals with and without laser irradiation.

Cytocompatibility and bone regeneration. Bone marrow mesenchymal stem cells (BMSCs) in the 3rd passage from 3-day SD rats were chosen to study the osteogenic behaviors of samples. The BMSCs were cultured with $\alpha$-MEM (HyClone, USA) with $10 \%$ fetal bovine serum (FBS) and $1 \%$ penicillin-streptomycin solution at $37{ }^{\circ} \mathrm{C}$ with 
$5 \% \mathrm{CO}_{2}$ for $1 \mathrm{~d}$. Confocal laser scanning microscopy (CLSM, OLYMPUS, Japan) was used for stained cell observation. Specifically, the cells were fixed with $4 \%$ paraformaldehyde, permeabilized with $0.1 \%$ Trion X-100 in PBS, stained with FITC-phalloidin (Solarbio, China) and DAPI (Solarbio, China), respectively. The cells were also fixed with $2.5 \%$ glutaraldehyde, dehydrated with graded concentration ethanol series $(10,30,50,70,90$ and $100 \mathrm{v} / \mathrm{v} \%)$, and gold sputtered for SEM observation. Additionally, dead and live cells were stained by live-dead cell staining kit (KeyGEN BioTECH, China), and observed by a fluorescent inverted microscope (OLYMPUS, Japan). The cell proliferation was investigated using 3-(4, 5-dimethylthiazol-2-yl)-2, 5-diphenyltetrazolium bromide (MTT) assay (Sigma-Aldrich, Shanghai) after culturing for 1, 3, 5 and 7 days, respectively.

Rabbits (6 8 months) weighted $2.5 \sim 3.0 \mathrm{~kg}$ was provided by Chengdu Dossy Experimental Animals Co., Ltd. Implant samples $(10 \mathrm{~mm} \times 5 \mathrm{~mm} \times 1 \mathrm{~mm})$ were inserted into the rabbit tibia for 3 weeks and 6 weeks respectively $(n=5)$. The extracted implants were immersed in $4 \%$ paraformaldehyde for $48 \mathrm{~h}$ and scanned by MicroCT (VivaCT 80, Switzerland) for cross-section images. Afterwards, the bone volume density (bone volume/total volume, BT/TV) was quantified.

Tumor inhibition experiments. Human osteosarcoma cell MG63 were obtained from American Type Culture Collection (ATCC). MG63 were cultured with samples (diameter: $6 \mathrm{~mm}$, height: $1 \mathrm{~mm}$ ) in 48-well plate with DMEM medium (HyClone, USA) with $10 \%$ fetal bovine serum (FBS) and 1\% penicillin-streptomycin solution at $37{ }^{\circ} \mathrm{C}$ under $5 \% \mathrm{CO}_{2}$ for $24 \mathrm{~h}$ [63]. The samples were then irradiated by $808 \mathrm{~nm}$ NIR 
laser under the power density of $0.31 \mathrm{~W} / \mathrm{cm}^{2}$ for $10 \mathrm{~min}$. The cell culture without laser irradiation was set as the control. After $12 \mathrm{~h}$ incubation, the morphology of the tumor cells with and without laser irradiation was observed by SEM (JSM-7500F, JEOL, Japan). Cells were immobilized with $2.5 \%$ glutaraldehyde, dehydrated with graded concentration ethanol series $(10,30,50,70,90$ and $100 \mathrm{v} / \mathrm{v} \%)$, and gold sputtered for SEM observation. The cell morphology was observed by confocal laser scanning microscopy (CLSM, OLYMPUS, Japan). The cytoskeleton and nuclei were stained with FITC-phalloidin (Solarbio, China) and DAPI (Solarbio, China), respectively. Dead and live cells were stained by live-dead cell staining kit (KeyGEN BioTECH, China) and observed using a fluorescent inverted microscope (OLYMPUS, Japan).

To quantify the photothermal conversion effect of the samples with laser irradiation on MG63 cells in vitro, MTT assay was conducted. $25 \mu \mathrm{L}$ MTT was added to each well and incubated for $4 \mathrm{~h}$. The samples were then transferred to another 48-well plate. DMSO was used to dissolve the methylthionin. The corresponding absorbance was monitored at $570 \mathrm{~nm}$ using a microplate reader (SpectraMax 190, Molecular Devices, America).

To investigate the performance of photothermal therapy in vivo, female BALB/c nude mice ( 4 6 weeks) weighed 19 25 g were provided by Chengdu Dossy Experimental Animals Co., Ltd, China. UMR106 cells, originally obtained from American Type Culture Collection (ATCC), was injected into the right back of mice with a concentration of $5 \times 10^{6}$ cells/ $0.1 \mathrm{ml}$ (each group: $n=5$ ). The volume of tumor which was raised to $\sim 300 \mathrm{~mm}^{3}$ prior to implantation of composite samples ( $\Phi 6 \mathrm{~mm} \times$ 
$1 \mathrm{~mm}$ ) [64]. The mice were treated daily by $0.31 \mathrm{~W} / \mathrm{cm}^{2}$ laser irradiation for $10 \mathrm{~min}$ for up to 14 days. The corresponding weight and tumor volume (V) were recorded on day $3,7,11$ and 14 . The tumor tissues on day 7 were then extracted from different groups to perform the H\&E staining.

Supporting Information. Brief statement in non-sentence format listing the contents of the material supplied as Supporting Information.

\section{Author information}

\section{Corresponding authors}

Li Zhang - Analytical \& Testing Center, Sichuan University, Chengdu 610065, China; E-mail: nic1976@ scu.edu.cn; ORCID: 0000-0002-0324-5092

\section{Author Contributions}

The manuscript was written through the contributions of all authors. All authors have approved the final version of the manuscript.

\section{Acknowledgement}

This work was funded by the National Natural Science Foundation of China (51673131) and International Collaborative Research Foundation of Sichuan Province (2018HH0068). We thank Prof. Xiaojun Wang for his advice on PEEK/G processing. We also thank TEM (Shanling Wang), XRD (Jiqiu Wen), ICP-OES (Xi Wu), MicroCT (Chenghui Li) and XPS (Shuguang Yan) groups of Analytical \& Testing Centre, Sichuan University for their assistance in testing.

\section{References}

[1]. Steven M. Kurtz, An Overview of PEEK Biomaterials. 2012, 1-7.

[2]. Lennert de Ruiter; Dennis Janssen; Adam Briscoe; Nico Verdonschot, A Preclinical Numerical Assessment of a Polyetheretherketone Femoral Component in Total Knee Arthroplasty during Gait. Journal of experimental orthopaedics 2017, 4 (1), 3-10.

[3]. KyoungTak Kang; YongGon Koh; Juhyun Son; Jin S. Yeom; Joon-Hee Park; 
Ho-Joong Kim, Biomechanical Evaluation of Pedicle Screw Fixation System in Spinal Adjacent Levels using Polyetheretherketone, Carbon-fiber-reinforced Polyetheretherketone, and Traditional Titanium as Rod Materials. Composites Part B: Engineering 2017, 130, 248-256.

[4]. V. Simon; C. Albon; S. Simon, Silver Release from Hydroxyapatite Self-assembling Calcium-phosphate Glasses. Journal of Non-Crystalline Solids 2008, 354 (15), 1751-1755.

[5]. N. T. Evans; F. B. Torstrick; C. S. Lee; K. M. Dupont; D. L. Safranski; W. A. Chang; A. E. Macedo; A. S. Lin; J. M. Boothby； D. C. Whittingslow； R. A. Carson; R. E. Guldberg; K. Gall, High-strength, Surface-porous Polyether-ether-ketone for Load-bearing Orthopedic Implants. Acta biomaterialia 2015, 13, 159-167.

[6]. K. H. Tan; C. K. Chua; K. F. Leong; C. M. Cheah; P. Cheang; M. S. Abu Bakar; S. W. Cha, Scaffold Development Using Selective Laser Sintering of Polyetheretherketone-hydroxyapatite Biocomposite Blends. Biomaterials 2003, 24 (18), 3115-3123.

[7]. Ana M. Díez-Pascual; Angel L. Díez-Vicente, Nano-TiO ${ }_{2}$ Reinforced PEEK/PEI Blends as Biomaterials for Load-Bearing Implant Applications. ACS applied materials \& interfaces 2015, 7 (9), 5561-5573.

[8]. M. S. Abu Bakar; M. H. W. Cheng; S. M. Tang; S. C. Yu; K. Liao; C. T. Tan; K. A. Khor; P. Cheang, Tensile properties, Tension-tension Fatigue and Biological Response of Polyetheretherketone-hydroxyapatite Composites for Load-bearing Orthopedic Implants. Biomaterials 2003, 24 (13), 2245-2250.

[9]. Miaomiao He; Xianchun Chen; Zhijun Guo; Xiaotao Qiu; Yutao Yang; Chengli Su; Nan Jiang; Yubao Li; Dan Sun; Li Zhang, Super Tough Graphene Oxide Reinforced Polyetheretherketone for Potential Hard Tissue Repair Applications. Composites Science and Technology 2019, 174, 194-201.

[10].Tao Lu; Jin Wen; Shi Qian; Huiliang Cao; Congqin Ning; Xiaoxia Pan; Xinquan Jiang; Xuanyong Liu; Paul K. Chu, Enhanced Osteointegration on Tantalum-implanted Polyetheretherketone Surface with Bone-like Elastic Modulus. Biomaterials 2015, 51, 173-183.

[11].Jin Wen; Tao Lu; Xiao Wang; Lianyi Xu; Qianju Wu; Hongya Pan; Donghui Wang; Xuanyong Liu; Xinquan Jiang, In Vitro and in Vivo Evaluation of Silicate-Coated Polyetheretherketone Fabricated by Electron Beam Evaporation. ACS applied materials \& interfaces 2016, 8 (21), 13197-13206.

[12]. Yuchen Zhu; Zhe Cao; Ying Peng; Liqiu Hu; Tankut Guney; Bin Tang, Facile Surface Modification Method for Synergistically Enhancing the Biocompatibility and Bioactivity of Poly(ether ether ketone) That Induced Osteodifferentiation. ACS applied materials \& interfaces 2019, 11 (31), 27503-27511.

[13].Taosheng Dong; Chunyan Duan; Song Wang; Xiangyu Gao; Qizhang Yang; Weizhong Yang; Yi Deng, Multifunctional Surface with Enhanced Angiogenesis for Improving Long-Term Osteogenic Fixation of Poly(ether ether ketone) Implants. ACS applied materials \& interfaces 2020, 12 (13), 14971-14982. 
[14]. Byung-Dong Hahn; Dong-Soo Park; Jong-Jin Choi; Jungho Ryu; Woon-Ha Yoon; Joon-Hwan Choi; Jong-Woo Kim; Cheol-Woo Ahn; Hyoun-Ee Kim; Byung-Ho Yoon; In-Kwon Jung, Osteoconductive Hydroxyapatite Coated PEEK for Spinal Fusion Surgery. Applied Surface Science 2013, 283, 6-11.

[15].John W. Durham; Sergio A. Montelongo; Joo L. Ong; Teja Guda; Matthew J. Allen; Afsaneh Rabiei, Hydroxyapatite Coating on PEEK Implants: Biomechanical and Histological Study in a Rabbit Model. Materials Science and Engineering: C 2016, 68, 723-731.

[16].Vinayaraj Ozhukil Kollath; Qiang Chen; Steven Mullens; Jan Luyten; Karl Traina; Aldo R. Boccaccini; Rudi Cloots, Electrophoretic Deposition of Hydroxyapatite and Hydroxyapatite-alginate on Rapid Prototyped 3D Ti6Al4V Scaffolds. Journal of Materials Science 2016, 51 (5), 2338-2346.

[17].Muhammad Atiq Ur Rehman; Fatih Erdem Bastan; Aneeqa Nawaz; Qaisar Nawaz; Abdul Wadood, Electrophoretic Deposition of PEEK/bioactive glass Composite Coatings on Stainless Steel for Orthopedic Applications: An optimization for In Vitro Bioactivity and Adhesion Strength. The International Journal of Advanced Manufacturing Technology 2020, 108 (5), 1849-1862.

[18].A. D'Elia; J. Deering; A. Clifford; B. E. J. Lee; K. Grandfield; I. Zhitomirsky, Electrophoretic Deposition of Polymethylmethacrylate and Composites for Biomedical Applications. Colloids and surfaces. B, Biointerfaces 2020, 188, 110763.

[19].Giang T. H. Tran; Masaki Koike; Tetsuo Uchikoshi; Hiroshi Fudouzi, Fabrication of Polystyrene Colloidal Crystal Film by Electrophoretic Deposition. Advanced Powder Technology 2020, 31 (8), 3085-3092.

[20].B. Mayoral; E. Harkin-Jones; P. Noorunnisa Khanam; M. A. AlMaadeed; M. Ouederni; A. R. Hamilton; D. Sun, Melt Processing and Characterisation of Polyamide 6/graphene Nanoplatelet Composites. RSC Advances 2015, 5 (65), 52395-52409.

[21].Jinglei Ping; Ramya Vishnubhotla; Amey Vrudhula; A. T. Charlie Johnson, Scalable Production of High-Sensitivity, Label-Free DNA Biosensors Based on Back-Gated Graphene Field Effect Transistors. ACS Nano 2016, 10 (9), 8700-8704.

[22].Tapas R. Nayak; Henrik Andersen; Venkata S. Makam; Clement Khaw; Sukang Bae; Xiangfan Xu; Pui-Lai R. Ee; Jong-Hyun Ahn; Byung Hee Hong; Giorgia Pastorin; Barbaros Özyilmaz, Graphene for Controlled and Accelerated Osteogenic Differentiation of Human Mesenchymal Stem Cells. ACS Nano 2011, 5 (6), 4670-4678.

[23].Da Huo; Ge Liu; Yanzhao Li; Yuxin Wang; Ge Guan; Mingcan Yang; Keyu Wei; Jingyuan Yang; Lingqin Zeng; Gang Li; Wen Zeng; Chuhong Zhu, Construction of Antithrombotic Tissue-Engineered Blood Vessel via Reduced Graphene Oxide Based Dual-Enzyme Biomimetic Cascade. ACS Nano 2017, 11 (11), 10964-10973.

[24].Hyunwoo Kim; Duhwan Lee; Jinhwan Kim; Tae-il Kim; Won Jong Kim, Photothermally Triggered Cytosolic Drug Delivery via Endosome Disruption 
Using a Functionalized Reduced Graphene Oxide. ACS Nano 2013, 7 (8), 6735-6746.

[25].E. Abbasi; A. Akbarzadeh; M. Kouhi; M. Milani, Graphene: Synthesis, Bio-applications, and Properties. Artificial Cells Nanomedicine and Biotechnology 2016, 44 (1), 150-156.

[26].P. Noorunnisa Khanam; M. A. AlMaadeed; M. Ouederni; Eileen Harkin-Jones; Beatriz Mayoral; Andrew Hamilton; Dan Sun, Melt processing and Properties of Linear Low Density Polyethylene-graphene Nanoplatelet Composites. Vacuum 2016, 130, 63-71.

[27].Cui Mao; Yutian Zhu; Wei Jiang, Design of Electrical Conductive Composites: Tuning the Morphology to Improve the Electrical Properties of Graphene Filled Immiscible Polymer Blends. ACS applied materials \& interfaces 2012, 4 (10), 5281-5286.

[28].P. Noorunnisa Khanam; Mariam A. AlMaadeed; M. Ouederni; Beatriz Mayoral; Andrew Hamilton; Dan Sun, Effect of Two Types of Graphene Nanoplatelets on The Physico-mechanical Properties of Linear Low-density Polyethylene Composites. Advanced Manufacturing: Polymer \& Composites Science 2016, 2 (2), 67-73.

[29]. Yafang Zhuang; Kun Zheng; Xinyu Cao; Qingrui Fan; Gang Ye; Jiaxin Lu; Jingnan Zhang; Yongmei Ma, Flexible Graphene Nanocomposites with Simultaneous Highly Anisotropic Thermal and Electrical Conductivities Prepared by Engineered Graphene with Flat Morphology. ACS Nano 2020, 14 (9), 11733-11742.

[30].Muhammad Khan; Edoardo Cantù; Sarah Tonello; Mauro Serpelloni; Nicola Lopomo; Emilio Sardini, A Review on Biomaterials for 3D Conductive Scaffolds for Stimulating and Monitoring Cellular Activities. Applied Sciences 2019, 9 (5), 271-292.

[31].Payam Baei; Mahya Hosseini; Hossein Baharvand; Sara Pahlavan, Electrically Conductive Materials for In Vitro Cardiac Microtissue Engineering. Journal of Biomedical Materials Research 2020, 108 (5), 1203-1213.

[32].Hongshi Ma; Chuan Jiang; Dong Zhai; Yongxiang Luo; Yu Chen; Fang Lv; Zhengfang Yi; Yuan Deng; Jinwu Wang; Jiang Chang; Chengtie Wu, A Bifunctional Biomaterial with Photothermal Effect for Tumor Therapy and Bone Regeneration. Advanced Functional Materials 2016, 26 (8), 1197-1208.

[33].Meng-Chin Wu; Archana R. Deokar; Jhan-Hong Liao; Po-Yuan Shih; Yong-Chien Ling, Graphene-Based Photothermal Agent for Rapid and Effective Killing of Bacteria. ACS Nano 2013, 7 (2), 1281-1290.

[34].He Wang; Baohua Zhao; Wenjing Dong; Yuan Zhong; Xiaorong Zhang; Yali Gong; Rixing Zhan; Malcolm Xing; Jianxiang Zhang; Gaoxing Luo; Wei Qian, A Dual-targeted Platform Based on Graphene for Synergistic Chemo-photothermal Therapy Against Multidrug-resistant Gram-negative bacteria and Their Biofilms. Chemical Engineering Journal 2020, 393, 124595.

[35].Bing Zhou; Xiangbo Ji; Ye Sheng; Lifeng Wang; Zhenhua Jiang, Mechanical and Thermal Properties of Polyetheretherketone Reinforced with $\mathrm{CaCO}_{3}$. 
European Polymer Journal 2004, 40 (10), 2357-2363.

[36].Beatriz Mayoral; Eileen Harkin-Jones; P Noorunnisa Khanam; MA AlMaadeed; Mabrouk Ouederni; AR Hamilton; Dan Sun, Melt Processing and Characterisation of Polyamide 6/graphene Nanoplatelet Composites. RSC Advances 2015, 5 (65), 52395-52409.

[37].Matthew Smith; Louis Scudiero; Juan Espinal; Jean-Sabin McEwen; Manuel Garcia-Perez, Improving the Deconvolution and Interpretation of XPS spectra from Chars by Ab Initio Calculations. Carbon 2016, 110, 155-171.

[38].E. Lewin; P. O. A Persson; M. Lattemann; M. Stüber; M. Gorgoi; A. Sandell; C. Ziebert; F. Schäfers; W. Braun; J. Halbritter; S. Ulrich; W. Eberhardt; L. Hultman; H. Siegbahn; S. Svensson; U. Jansson, On the origin of a third spectral component of C1s XPS-spectra for nc-TiC/a-C Nanocomposite Thin Films. Surface and Coatings Technology 2008, 202 (15), 3563-3570.

[39].Shengnan Wang; Yi Deng; Lei Yang; Xiuyuan Shi; Weizhong Yang; Zhi-Gang Chen, Enhanced Antibacterial Property and Osteo-differentiation Activity on Plasma Treated Porous Polyetheretherketone with Hierarchical micro/nano-topography. Journal of Biomaterials Science, Polymer Edition 2018, 29 (5), 520-542.

[40].Dirk Rosenthal; Marina Ruta; Robert Schlögl; Lioubov Kiwi-Minsker, Combined XPS and TPD Study of Oxygen-functionalized Carbon Nanofibers Grown on Sintered Metal Fibers. Carbon 2010, 48 (6), 1835-1843.

[41].Limei Li; Yi Zuo; Qin Zou; Boyuan Yang; Lili Lin; Jidong Li; Yubao Li, Hierarchical Structure and Mechanical Improvement of an n-HA/GCO-PU Composite Scaffold for Bone Regeneration. ACS applied materials \& interfaces 2015, 7 (40), 22618-22629.

[42].Li Yubao; C. P. A. T. Klein; Zhang Xingdong; K. de Groot, Formation of a Bone Apatite-like Layer on The Surface of Porous Hydroxyapatite Ceramics. Biomaterials 1994, 15 (10), 835-841.

[43].Zhang Li; Li Yubao; Yang Aiping; Peng Xuelin; Wang Xuejiang; Zhang Xiang, Preparation and In Vitro Investigation of Chitosan/nano-hydroxyapatite Composite Used as Bone Substitute Materials. Journal of Materials Science: Materials in Medicine 2005, 16 (3), 213-219.

[44].E. Mohseni; E. Zalnezhad; A. R. Bushroa, Comparative Investigation on the Adhesion of Hydroxyapatite Coating on Ti-6Al-4V Implant: A review paper. International Journal of Adhesion and Adhesives 2014, 48, 238-257.

[45].Farbod Sharif; Ali Shayesteh Zeraati; Pejman Ganjeh-Anzabi; Nael Yasri; Maria Perez-Page; Stuart M. Holmes; Uttandaraman Sundararaj; Milana Trifkovic; Edward P. L. Roberts, Synthesis of a High-temperature Stable Electrochemically Exfoliated Graphene. Carbon 2020, 157, 681-692.

[46].Hamra Assyaima Abdul Bashid; Hong Ngee Lim; Sazlinda Kamaruzaman; Suraya Abdul Rashid; Robiah Yunus; Nay Ming Huang; Chun Yang Yin; Mohammad Mahbubur Rahman; Mohammednoor Altarawneh; Zhong Tao Jiang; Pandikumar Alagarsamy, Electrodeposition of Polypyrrole and Reduced Graphene Oxide onto Carbon Bundle Fibre as Electrode for Supercapacitor. 
Nanoscale Research Letters 2017, 12 (1), 246.

[47].J. van Tassel; C. Randall, Potential for Integration of Electrophoretic Deposition into Electronic Device Manufacture; Demonstrations Using Silver/palladium. Journal of Materials Science 2004, 39 (3), 867-879.

[48].Gerd Teike; Mathias Dietzel; Burkhard Michaelis; Helmut Schomburg; Martin Sommerfeld, Multiscale Lattice-Boltzmann Approach for Electrophoretic Particle Deposition. Aerosol Science and Technology 2012, 46 (4), 451-464.

[49].Subhadip Basu; Biswajit Gorai; Bikramjit Basu; Prabal K. Maiti, Electric Field Mediated Fibronectin-hydroxyapatite Interaction: A Molecular Insight. 2020, 2020.07.22.215327.

[50].Yu-Wei Chen; Yu-Lin Su; Shang-Hsiu Hu; San-Yuan Chen, Functionalized Graphene Nanocomposites for Enhancing Photothermal Therapy in Tumor Treatment. Advanced Drug Delivery Reviews 2016, 105, 190-204.

[51]. Yuan Li; Xiangmei Liu; Bo Li; Yufeng Zheng; Yong Han; Da-fu Chen; Kelvin Wai Kwok Yeung; Zhenduo Cui; Yanqin Liang; Zhaoyang Li; Shengli Zhu; Xianbao Wang; Shuilin Wu, Near-Infrared Light Triggered Phototherapy and Immunotherapy for Elimination of Methicillin-Resistant Staphylococcus aureus Biofilm Infection on Bone Implant. ACS Nano 2020, 14 (7), 8157-8170.

[52]. Vincent Lee; Robert V. Dennis; Brian J. Schultz; Cherno Jaye; Daniel A. Fischer; Sarbajit Banerjee, Soft X-ray Absorption Spectroscopy Studies of the Electronic Structure Recovery of Graphene Oxide upon Chemical Defunctionalization. The Journal of Physical Chemistry C 2012, 116 (38), 20591-20599.

[53].Hongye Yao; Yang Huang; Xuan Li; Xuehua Li; Hongbin Xie; Tianlie Luo; Jingwen Chen; Zhongfang Chen, Underlying Mechanisms of Reactive Oxygen Species and Oxidative Stress Photoinduced by Graphene and Its Surface-functionalized Derivatives. Environmental Science: Nano 2020, 7 (3), 782-792.

[54].Mohammad Yousef Memar; Reza Ghotaslou; Mohammad Samiei; Khosro Adibkia, Antimicrobial Use of Reactive Oxygen Therapy: Current Insights. Infect Drug Resist 2018, 11, 567-576.

[55].Tanveer A. Tabish; Shaowei Zhang; Paul G. Winyard, Developing the Next Generation of Graphene-based Platforms for Cancer Therapeutics: The potential Role of Reactive Oxygen Species. Redox Biology 2018, 15, 34-40.

[56].Y. C. Chai; A. Carlier; J. Bolander; S. J. Roberts; L. Geris; J. Schrooten; H. Van Oosterwyck; F. P. Luyten, Current Views on Calcium Phosphate Osteogenicity and the Translation into Effective Bone Regeneration Strategies. Acta biomaterialia 2012, 8 (11), 3876-3887.

[57].Song Zhou; Yu-Bao Li; Yos S. Morsi; Yan-Ying Wang; Chen Chen; Li Zhang, Effects of Ethylene-Vinyl Acetate Copolymer on the Morphology and Mechanical Properties of Hydroxyapatite/Polyamide 66 Composites for Bone Tissue Engineering. Polymer-Plastics Technology and Engineering 2014, 53 (3), 290-297. 
[58].Jie Wei; Yubao Li, Tissue Engineering Scaffold Material of Nano-apatite Crystals and Polyamide Composite. European Polymer Journal 2004, 40 (3), 509-515.

[59].S. A. Bender; J. D. Bumgardner; M. D. Roach; K. Bessho; J. L. Ong, Effect of Protein on the Dissolution of HA Coatings. Biomaterials 2000, 21 (3), 299-305.

[60].Jianhua Lv; Jing Jin; Jiayue Chen; Bing Cai; Wei Jiang, Antifouling and Antibacterial Properties Constructed by Quaternary Ammonium and Benzyl Ester Derived from Lysine Methacrylamide. ACS applied materials \& interfaces 2019, 11 (28), 25556-25568.

[61].H. Chen; G. Lan; L. Ran; Y. Xiao; K. Yu; B. Lu; F. Dai; D. Wu; F. Lu, A Novel Wound Dressing Based on a Konjac Glucomannan/silver Nanoparticle Composite Sponge Effectively Kills Bacteria and Accelerates Wound Healing. Carbohydrate Polymers 2018, 183, 70-80.

[62].Junyong Zhu; Jingwei Hou; Yatao Zhang; Miaomiao Tian; Tao He; Jindun Liu; Vicki Chen, Polymeric Antimicrobial Membranes Enabled by Nanomaterials for Water Treatment. Journal of Membrane Science. 2018, 550, 173-197.

[63]. Qian Chen; Ligeng Xu; Chao Liang; Chao Wang; Rui Peng, Photothermal Therapy with Immune-adjuvant Nanoparticles Together with Checkpoint Blockade for Effective Cancer Immunotherapy. Nature Communications 2016, 7 , 13193.

[64].Ning Ma; Ming-Kang Zhang; Xiao-Shuang Wang; Lu Zhang; Jun Feng; Xian-Zheng Zhang, NIR Light-Triggered Degradable MoTe 2 Nanosheets for Combined Photothermal and Chemotherapy of Cancer. Advanced Functional Materials 2018, 28 (31), 1801139.
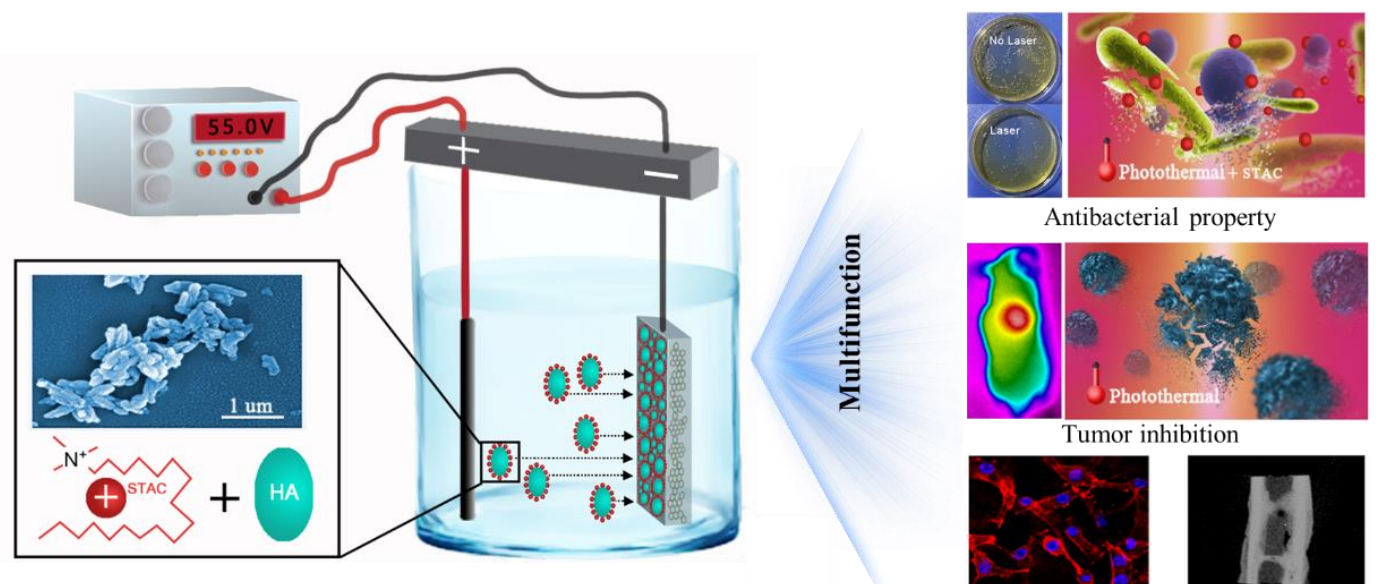

Antibacterial property
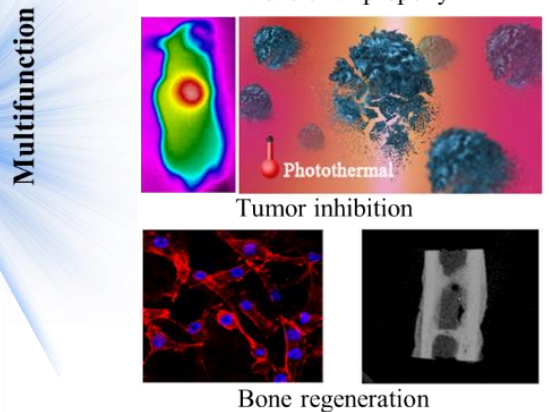

TOC graphic 